\title{
One-pot synthesis and UV-Visible absorption studies of novel tricyclic heterocycle tethered Xanthene-1,8-diones
}

\author{
THIRUMAL YEMPALA ${ }^{\mathrm{a}}$, BALASUBRAMANIAN SRIDHAR ${ }^{\mathrm{b}}$ and SRINIVAS KANTEVARI ${ }^{\mathrm{a}, *}$ \\ ${ }^{a}$ CPC Division (Organic Chemistry Division-II), CSIR-Indian Institute of Chemical Technology, \\ Hyderabad 500 007, India \\ ${ }^{\mathrm{b}}$ Centre for X-ray Crystallography, CSIR-Indian Institute of Chemical Technology, Hyderabad 500 007, India \\ e-mail: kantevari@yahoo.com; kantevari@gmail.com
}

MS received 07 July 2014; revised 28 December 2014; accepted 29 December 2014

\begin{abstract}
A series of new tricyclic heterocyclic xanthene-1,8-diones tethered with chromophoric dibenzo $[b, d]$ furan, dibenzo $[b, d]$ thiophene and 9-methyl-9H-carbazoles were synthesized through one-pot condensation of dibenzo $[b, d]$ furan-2-carbaldehyde, dibenzo $[b, d]$ thiophene-2-carbaldehyde and 9-methyl-9Hcarbazole-3-carbaldehyde with cyclic 1,3-dicarbonyls in the presence of recyclable $\mathrm{PPA}_{-} \mathrm{SiO}_{2}$ catalyst under solvent-free conditions. Further, UV-Visible absorption properties of all the synthesized compounds were investigated in $\mathrm{CHCl}_{3}$, THF and acetonitrile.
\end{abstract}

Keywords. Xanthenediones; Dibenzo[ $b, d]$ furan; 9-Methyl carbazole; Dibenzo[ $b, d]$ thiophene; One-pot synthesis; UV-Visible absorption studies.

\section{Introduction}

In recent years xanthene derivatives have attracted scientists world over due to their promising applications in medicinal and material chemistry. ${ }^{1-5}$ Among this class, xanthenediones play a vital role as the key structural unit in a variety of natural products exhibiting several biological activities. ${ }^{3-5}$ Due to the presence of in-built pyran ring architecture and their extended conjugation with aromatic groups, xanthenediones exhibit applications in optoelectronic materials. They form an important component in laser dyes ${ }^{6}$ and are used in laser technologies $^{7}$ for photodynamic therapy. Xanthenediones are also used as $\mathrm{pH}$ sensitive fluorescent materials for visualization of biomolecules. ${ }^{8}$ In view of the importance of xanthenediones, we would like to extend the conjugation within the xanthenedione moiety by replacing the simple/substituted phenyl groups with tricyclic heterocycles.

For the past decade, tricyclic systems such as dibenzofuran, dibenzothiophene and carbazole with planar architecture $^{9 \mathrm{a}}$ have got significant interest in the area of materials, dyes, photochemistry, and light-emitting diodes. They also act as ideal conjugated units for the construction of organic semiconductors. Among these three heterocycles, dibenzofuran has been used as an effective organic material for phosphorescent organic

\footnotetext{
*For correspondence
}

light-emitting diodes and electron-transport materials or electron-transport-type host materials because of its high triplet energy and strong electron-withdrawing oxygen linkage in its molecular structure. ${ }^{9 \mathrm{~b}-\mathrm{c}}$ Moreover, all these heterocycles dibenzofuran, carbazole and benzothiophene are naturally abundant with more pronounced anti-bacterial, anti-depressant, and antituberculosis activities. ${ }^{10 a-d}$

\section{Experimental}

\subsection{General remarks}

Melting points were measured with a Fischer-Johns melting point apparatus and are uncorrected. IR spectra were recorded as $\mathrm{KBr}$ pellets and absorptions are reported in $\mathrm{cm}^{-1}$. NMR spectra were recorded on 300 (Bruker) and $500 \mathrm{MHz}$ (Varian) spectrometers in appropriate solvents using TMS as internal standard and the chemical shifts are shown in $\delta$ scales. ${ }^{13} \mathrm{C}$ NMR spectra were recorded on $75 \mathrm{MHz}$ spectrometers. Highresolution mass spectra were obtained by using ESIQTOF mass spectrometry. All the experiments were monitored by analytical thin layer chromatography (TLC) performed on silica gel GF254 pre-coated plates. After elution, the plate was visualized under UV illumination at $254 \mathrm{~nm}$ for UV active materials. Silica gel finer than 200 mesh was used for column chromatography. Yields refer to chromatographically and 
spectroscopically homogeneous materials, unless otherwise stated. Appropriate names for all the new compounds were given with the help of ChemBioOffice 12.0; 2010. All the photophysical properties for these compounds were recorded with JASCO 500 UV Spectrophotometer. All the graphs were drawn using Origin software version 7.0. The solvents for column elution were purchased commercially and for UV-visible studies the solvents were purchased from Sigma Aldrich.

\subsection{General procedure for the synthesis of aldehydes} $2 a, 2 b$ and $2 c$

All these aldehydes were synthesized according to the procedure described in literature. ${ }^{11 \text { a }}$ Analytical data is in agreement with the literature data for dibenzo $[b, d]$ furan-2-carbaldehyde (2a), ${ }^{11}$ a 9 -methyl- $9 \mathrm{H}$-carbazole3 -carbaldehydes $(\mathbf{2 b})^{11 \mathrm{a}}$ and dibenzo $[b, d]$ thiophene-2carbaldehyde (2c). ${ }^{9}$

Spectral data of compound $2 c$ : Light yellow solid; yield: $60 \%$; M.p. $61-63^{\circ} \mathrm{C} .{ }^{1} \mathrm{H}$ NMR $(300 \mathrm{MHz}$, $\left.\mathrm{CDCl}_{3}\right): \delta 10.13(\mathrm{~s}, 1 \mathrm{H}), 8.59(\mathrm{t}, J=0.7 \& 1.3 \mathrm{~Hz}, 1 \mathrm{H})$, 8.27-8.13 (m, $1 \mathrm{H}), 8.00-7.90$ (m, $2 \mathrm{H}), 7.89-7.82$ (m, $1 \mathrm{H}), 7.55-7.43(\mathrm{~m}, 2 \mathrm{H})$.

\subsection{General procedure for the synthesis of Xanthenediones}

A mixture of aldehyde $(1 \mathrm{mmol})$, diketone $(2 \mathrm{mmol})$ and $\mathrm{PPA}-\mathrm{SiO}_{2}(20 \mathrm{~mol} \%)$ were heated at $125^{\circ} \mathrm{C}$ with stirring for the time specified in table 2. After completion, the reaction mixture was cooled to room temperature, and dichloromethane was added and the catalyst was filtered off. The filtrate thus obtained was concentrated under vacuum. Recrystalization of crude mass in $\mathrm{CHCl}_{3}$ : hexane (1:5) yielded pure xanthenediones $\mathbf{6 , 7}$ $\& \mathbf{8}$ as crystalline solids.

2.3a 9-(Dibenzo[b,d]furan-2-yl)-3,4,5,6,7,9-hexahydro1H-xanthene-1,8(2H)-dione ( $6 a$ ): Yellow solid; yield: $364 \mathrm{mg}$ (95\%); M.p. $198-200^{\circ} \mathrm{C}$. IR (KBr): 2925,1672 , 1621, 1478, 1430, 1359, 1200, 1176, 1129, 1010, 956, $755 \mathrm{~cm}^{-1} .{ }^{1} \mathrm{H}$ NMR $\left(300 \mathrm{MHz}, \mathrm{CDCl}_{3}\right): \delta 7.95(\mathrm{~d}, J=$ $7.7 \mathrm{~Hz}, 1 \mathrm{H}), 7.86(\mathrm{~d}, J=1.5 \mathrm{~Hz}, 1 \mathrm{H}), 7.45(\mathrm{~d}, J=$ $8.1 \mathrm{~Hz}, 1 \mathrm{H}), 7.40-7.32(\mathrm{~m}, 2 \mathrm{H}), 7.31-7.22(\mathrm{~m}, 2 \mathrm{H})$, $4.91(\mathrm{~s}, 1 \mathrm{H}), 2.74-2.50(\mathrm{~m}, 4 \mathrm{H}), 2.40-2.23(\mathrm{~m}, 4 \mathrm{H})$, 2.11-1.94 (m, $4 \mathrm{H}) .{ }^{13} \mathrm{C}$ NMR $\left(75 \mathrm{MHz}, \mathrm{CDCl}_{3}\right): \delta$ 196.6, 163.8, 156.4, 154.9, 139.1, 127.4, 126.8, 123.9, 123.6, 122.4, 120.8, 120.7, 117.1, 111.4, 111.1, 36.9, 31.6, 27.1, 20.2. HRMS (ESI): $m / z[\mathrm{M}+\mathrm{H}]^{+}$calcd for $\mathrm{C}_{25} \mathrm{H}_{21} \mathrm{O}_{4}$ : 385.1439; found: 385.1446. 2.3b 9-(Dibenzo[b,d]furan-2-yl)-3,3,6,6-tetramethyl-3, 4,5,6,7,9-hexahydro-1H-xanthene-1,8(2H)-dione (7a): White solid; yield: $404 \mathrm{mg}$ (92\%); M.p. 190-195 ${ }^{\circ} \mathrm{C}$. IR (KBr): 2955, 2927, 1661, 1622, 1448, 1431, 1360, $1200,1166,1138,1002,746 \mathrm{~cm}^{-1} .{ }^{1} \mathrm{H}$ NMR $(300 \mathrm{MHz}$, $\left.\mathrm{CDCl}_{3}\right): \delta 7.94(\mathrm{~d}, J=6.9 \mathrm{~Hz}, 1 \mathrm{H}), 7.88(\mathrm{~d}, J=1.7 \mathrm{~Hz}$, $1 \mathrm{H}), 7.46(\mathrm{~d}, J=8.1 \mathrm{~Hz}, 1 \mathrm{H}), 7.41-7.31(\mathrm{~m}, 2 \mathrm{H})$, 7.29-7.22 (m, $2 \mathrm{H}), 4.86(\mathrm{~s}, 1 \mathrm{H}), 2.48(\mathrm{~s}, 4 \mathrm{H})$, 2.25-2.10 (m, $4 \mathrm{H}), 1.12(\mathrm{~s}, 6 \mathrm{H}), 1.01(\mathrm{~s}, 6 \mathrm{H}) .{ }^{13} \mathrm{C}$ NMR $\left(75 \mathrm{MHz}, \mathrm{CDCl}_{3}\right): \delta 196.4,162.1,156.4,154.8$, $138.8,127.2$, 126.7 123.5, 122.4, 120.8, 115.8, 111.3, 111.0, 50.7, 40.8, 31.7, 29.2, 27.2. HRMS (ESI): $\mathrm{m} / \mathrm{z}$ $[\mathrm{M}+\mathrm{Na}]^{+}$calcd for $\mathrm{C}_{29} \mathrm{H}_{28} \mathrm{O}_{4} \mathrm{Na}$ : 463.1885; found: 463.1887 .

2.3c 9-(Dibenzo[b,d]furan-2-yl)-3,6-diisopropyl-3,4,5, 6,7,9-hexahydro-1H-xanthene-1,8(2H)-dione (8a): Pale yellow solid; yield: $439 \mathrm{mg}$ (94\%); M.p. $200-205^{\circ} \mathrm{C}$. IR (KBr): 2960, 2874, 1674, 1623, 1476, 1448, 1371, 1353, 1243, 1189, 1136, 1023, 989, 841, $761 \mathrm{~cm}^{-1}$. ${ }^{1} \mathrm{H}$ NMR $\left(300 \mathrm{MHz}, \mathrm{CDCl}_{3}\right): \delta 8.00-7.91(\mathrm{~m}, 1 \mathrm{H})$, $7.88-7.78(\mathrm{dd}, J=8.8 \mathrm{~Hz} \& 1.7 \mathrm{~Hz}, 1 \mathrm{H}), 7.46(\mathrm{~d}, J=$ $7.9 \mathrm{~Hz}, 1 \mathrm{H}), 7.42-7.31(\mathrm{~m}, 2 \mathrm{H}), 7.30-7.21(\mathrm{~m}, 2 \mathrm{H})$, $4.87(\mathrm{~d}, J=3.0 \mathrm{~Hz}, 1 \mathrm{H}), 2.71-2.27(\mathrm{~m}, 6 \mathrm{H}), 2.17-1.80$ (m, $4 \mathrm{H}), 1.70-1.52(\mathrm{~m}, 2 \mathrm{H}), 1.04-0.90(\mathrm{~m}, 12 \mathrm{H})$. ${ }^{13} \mathrm{C}$ NMR $\left(75 \mathrm{MHz}, \mathrm{CDCl}_{3}\right): \delta 196.9,163.5,156.3$, $154.8,138.9,127.4,126.7,124.2,123.8,122.3,120.8$, 116.6, 116.3, 111.3, 111.0, 41.3, 40.8, 38.7, 31.7, 30.8, 19.4. HRMS (ESI): $m / z[\mathrm{M}+\mathrm{H}]^{+}$calcd for $\mathrm{C}_{31} \mathrm{H}_{33} \mathrm{O}_{4}$ : 469.2378; found: 469.2361 .

2.3d 9-(9-Methyl-9H-carbazol-3-yl)-3,4,5,6,7,9-hexahydro1H-xanthene-1,8(2H)-dione $(\boldsymbol{6} \boldsymbol{b})$ : White solid; yield: $361 \mathrm{mg}(91 \%)$; M.p. $190-195^{\circ} \mathrm{C}$. IR (KBr): 2926, 1734 , 1668, 1622, 1432, 1358, 1201, 1177, 1131, 1009, 959, $765,700 \mathrm{~cm}^{-1} .{ }^{1} \mathrm{H}$ NMR $\left(300 \mathrm{MHz}, \mathrm{CDCl}_{3}\right): \delta 8.04(\mathrm{~d}$, $J=8.3 \mathrm{~Hz}, 1 \mathrm{H}), 7.85(\mathrm{~s}, 1 \mathrm{H}), 7.41(\mathrm{~d}, J=8.3 \mathrm{~Hz}, 1 \mathrm{H})$, $7.34(\mathrm{t}, J=8.3 \mathrm{~Hz}, 1 \mathrm{H}), 7.28-7.22(\mathrm{~m}, 1 \mathrm{H}), 7.21-7.16$ $(\mathrm{d}, J=8.3 \mathrm{~Hz}, 1 \mathrm{H}), 7.12(\mathrm{t}, J=7.2 \mathrm{~Hz}, 1 \mathrm{H}), 4.92(\mathrm{~s}, 1 \mathrm{H})$, 3.77 (s, $3 \mathrm{H}), 2.74-2.51$ (m, $4 \mathrm{H}), 2.45-2.26$ (m, $4 \mathrm{H})$, 2.06-1.97 (m, $4 \mathrm{H}) .{ }^{13} \mathrm{C}$ NMR $\left(75 \mathrm{MHz}, \mathrm{CDCl}_{3}\right): \delta$ 196.8, 163.6, 141.2, 135.4, 126.6, 125.4, 122.7, 122.5, $120.4,119.9,118.5,117.5,108.2,107.9,37.0,31.5$, 29.0, 27.2, 20.3. HRMS (ESI): $m / z$ [M+Na $]^{+}$calcd for $\mathrm{C}_{26} \mathrm{H}_{23} \mathrm{O}_{3} \mathrm{NNa}$ : 420.1575; found: 420.1570.

$2.3 e \quad 3,3,6,6-T e t r a m e t h y l-9-(9-m e t h y l-9 H-c a r b a z o l-3-$ yl)-3,4,5,6,7,9-hexahydro-1H-xanthene-1,8 (2H)-dione (7b): Brown solid; yield: $421 \mathrm{mg}$ (93\%); M.p. 220$223^{\circ} \mathrm{C}$. IR (KBr): 3324, 2968, 2918, 1701, 1612, 1598, 1513, 1452, 1283, 1164, 1127, 1015, 836, $694 \mathrm{~cm}^{-1}$. 
${ }^{1} \mathrm{H}$ NMR $\left(300 \mathrm{MHz}, \mathrm{CDCl}_{3}\right): \delta 8.02(\mathrm{~d}, J=7.7 \mathrm{~Hz}, 1 \mathrm{H})$, 7.86 (s, $1 \mathrm{H}), 7.46-7.29$ (m, $2 \mathrm{H}), 7.28-7.17$ (m, $2 \mathrm{H})$, $7.11(\mathrm{t}, J=8.1 \mathrm{~Hz}, 1 \mathrm{H}), 4.87(\mathrm{~s}, 1 \mathrm{H}), 3.79(\mathrm{~s}, 3 \mathrm{H})$, $2.48(\mathrm{~s}, 4 \mathrm{H}), 2.28-2.07(\mathrm{~m}, 4 \mathrm{H}), 1.12(\mathrm{~s}, 6 \mathrm{H}), 1.00$ $(\mathrm{s}, 6 \mathrm{H}) .{ }^{13} \mathrm{C} \mathrm{NMR}\left(75 \mathrm{MHz}, \mathrm{CDCl}_{3}\right): \delta 196.5,161.8$, $141.1,139.81,135.17,126.6,125.2,122.7,120.2$, $119.8,118.4,116.2,108.2,107.8,50.7,40.8,32.2$, 31.6, 29.2, 27.3. HRMS (ESI): $m / z[\mathrm{M}+\mathrm{Na}]^{+}$calcd for $\mathrm{C}_{30} \mathrm{H}_{31} \mathrm{O}_{3} \mathrm{NNa}$ : 476.2201; found: 476.2196.

$2.3 \mathrm{f}$ 3,6-Diisopropyl-9-(9-methyl-9H-carbazol-3-yl)3,4,5,6,7,9-hexahydro-1H-xanthene-1,8(2H)-dione $(\mathbf{8 b})$ : Black solid; yield: $442 \mathrm{mg}$ (92\%); M.p. $120-124^{\circ} \mathrm{C}$. IR (KBr): 2958, 1662, 1492, 1471, 1370, 1186, 1138, $744 \mathrm{~cm}^{-1} .{ }^{1} \mathrm{H}$ NMR $\left(300 \mathrm{MHz}, \mathrm{CDCl}_{3}\right): \delta 8.08-8.00$ $(\mathrm{m}, 1 \mathrm{H}), 7.85-7.75(\mathrm{~m}, 1 \mathrm{H}), 7.45-7.29(\mathrm{~m}, 2 \mathrm{H})$, 7.27-7.07 (m, $3 \mathrm{H}), 4.87(\mathrm{~d}, J=4.1 \mathrm{~Hz}, 1 \mathrm{H}), 3.78$ $(\mathrm{s}, 3 \mathrm{H}), 2.84-2.21(\mathrm{~m}, 6 \mathrm{H}), 2.16-1.75(\mathrm{~m}, 4 \mathrm{H})$, 1.69-1.49 (m, $2 \mathrm{H}), 1.02-0.86(\mathrm{~m}, 12 \mathrm{H}) .{ }^{13} \mathrm{C}$ NMR $\left(75 \mathrm{MHz}, \mathrm{CDCl}_{3}\right): \delta 197.0,163.9,141.2,138.0$, $133.9,126.7,126.6,125.3,120.4,120.3,119.8,118.4$, $117.1,108.2,107.9,41.5,40.9,38.8,31.8,31.1$, 29.7, 19.4. HRMS (ESI): $m / z[\mathrm{M}+\mathrm{Na}]^{+}$calcd for $\mathrm{C}_{32} \mathrm{H}_{35} \mathrm{O}_{3} \mathrm{NNa}$ :504.2514; found: 504.2525.

\section{3g 9-(Dibenzo[b,d]thiophen-2-yl)-3,4,5,6,7,9-hexahydro-} $1 H$-xanthene-1,8(2H)-dione $(\boldsymbol{6 c})$ : White solid; yield: $372 \mathrm{mg}$ (93\%); M.p. $210-213^{\circ} \mathrm{C}$. IR (KBr): 2926, 1734, 1668, 1622, 1432, 1358, 1201, 1177, 1131, 1009, 959, $765,700 \mathrm{~cm}^{-1} .{ }^{1} \mathrm{H}$ NMR $\left(300 \mathrm{MHz}, \mathrm{CDCl}_{3}\right): \delta 8.20$ $8.14(\mathrm{~m}, 1 \mathrm{H}), 8.06(\mathrm{~d}, J=1.5 \mathrm{~Hz}, 1 \mathrm{H}), 7.78-7.71(\mathrm{~m}, 1 \mathrm{H})$, 7.69-7.63 (m, 1 H), 7.42-7.32 (m, $2 \mathrm{H}), 7.31-7.26$ (m, $1 \mathrm{H}), 4.93$ (s, $1 \mathrm{H}), 2.74-2.49$ (m, $4 \mathrm{H}), 2.40-2.22(\mathrm{~m}$, $4 \mathrm{H}), 2.11-1.91$ (m, $4 \mathrm{H}) .{ }^{13} \mathrm{C} \mathrm{NMR}\left(75 \mathrm{MHz}, \mathrm{CDCl}_{3}\right)$ : $\delta 196.5,163.9,140.9,139.6,137.4,135.4,129.9$, $127.1,126.3,124.0,122.5,122.3,121.6,116.8,36.8$, 31.6, 27.0, 20.2. HRMS (ESI): $m / z[\mathrm{M}+\mathrm{H}]^{+}$calcd for $\mathrm{C}_{25} \mathrm{H}_{21} \mathrm{O}_{3} \mathrm{~S}$ : 401.1211 ; found: 401.1206 .

2.3h 9-(Dibenzo[b,d]thiophen-2-yl)-3,3,6,6-tetramethyl3,4,5,6,7,9-hexahydro-1H-xanthene-1,8 (2H)-dione (7c): Pale yellow solid; yield: $414 \mathrm{mg}(91 \%)$; M.p. 200$204^{\circ} \mathrm{C}$. IR (KBr): 2957, 2916, 1656, 1621, 1464, 1429 , 1359, 1200, 1165, 1136, 1003, 763, 735 $\mathrm{cm}^{-1}$. ${ }^{1} \mathrm{H}$ NMR $\left(300 \mathrm{MHz}, \mathrm{CDCl}_{3}\right): \delta 8.19(\mathrm{~m}, 1 \mathrm{H}), 8.07(\mathrm{~d}, J=1.3 \mathrm{~Hz}$, $1 \mathrm{H}), 7.80-7.70(\mathrm{~m}, 1 \mathrm{H}), 7.69-7.62(\mathrm{~d}, J=8.3 \mathrm{~Hz}$, 1H), 7.42-7.26 (m, $3 \mathrm{H}), 4.88(\mathrm{~s}, 1 \mathrm{H}), 2.48$ (s, $4 \mathrm{H})$, 2.25-2.08 (ab d, $4 \mathrm{H}), 1.12$ (s, $6 \mathrm{H}), 1.00$ (s, $6 \mathrm{H}) .{ }^{13} \mathrm{C}$ NMR $\left(75 \mathrm{MHz}, \mathrm{CDCl}_{3}\right): \delta 196.4,162.2,140.6,139.6$, 135.4, 129.4, 127.1, 126.3, 124.0, 122.6, 122.3, 121.7, 121.6, 115.6, 50.6, 40.8, 31.8, 29.2, 27.2. HRMS (ESI): $m / z[\mathrm{M}+\mathrm{H}]^{+}$calcd for $\mathrm{C}_{29} \mathrm{H}_{29} \mathrm{O}_{3} \mathrm{~S}: 457.1837$; found: 457.1845 .
$2.3 \mathrm{i}$ 9-(Dibenzo[b,d]thiophen-2-yl)-3,6-diisopropyl-3, 4,5,6,7,9-hexahydro-1H-xanthene-1,8 (2H)-dione $(8 \mathrm{c})$ : Brick red solid; yield: $454 \mathrm{mg}(94 \%)$; M.p. $80-85^{\circ} \mathrm{C}$. IR (KBr): 2958, 1670, 1624, 1466, 1430, 1368, 1185 , $1137,819,762,733 \mathrm{~cm}^{-1} .{ }^{1} \mathrm{H}$ NMR $\left(300 \mathrm{MHz}, \mathrm{CDCl}_{3}\right)$ : $\delta 8.16(\mathrm{~s}, 1 \mathrm{H}), 8.05(\mathrm{~d}, J=10.7 \mathrm{~Hz}, 1 \mathrm{H}), 7.74(\mathrm{~d}, J=8.1 \mathrm{~Hz}$, $1 \mathrm{H}), 7.69-7.63(\mathrm{~m}, 1 \mathrm{H}), 7.46-7.17(\mathrm{~m}, 3 \mathrm{H}), 4.88(\mathrm{~s}, 1 \mathrm{H})$, 2.73-2.20 (m, $6 \mathrm{H}), 2.17-1.76(\mathrm{~m}, 4 \mathrm{H}), 1.68-1.42(\mathrm{~m}$, $2 \mathrm{H}), 1.04-0.75(\mathrm{~m}, 12 \mathrm{H}) .{ }^{13} \mathrm{C} \mathrm{NMR}\left(75 \mathrm{MHz}, \mathrm{CDCl}_{3}\right)$ : $\delta$ 196.8, 164.2, 140.7, 139.6, 137.5, 135.4, 129.1, 127.1, 127.0, 126.3, 124.0, 122.5, 122.3, 121.7, 121.6, 116.1, 41.3, 40.8, 38.7, 31.7, 31.0, 19.4. HRMS (ESI): $\mathrm{m} / \mathrm{z}$ $[\mathrm{M}+\mathrm{Na}]^{+}$calcd for $\mathrm{C}_{31} \mathrm{H}_{32} \mathrm{O}_{2} \mathrm{SNa}$ : 507.1969; found: 507.1995.

\section{Results and Discussion}

Inspired by the applications of dibenzo[ $b, d]$ furan (1a), 9-methyl-9H-carbazole (1b), dibenzo[b,d]thiophene (1c), and xanthenediones we envisaged to combine xanthenedione and tricyclic heterocycles in one molecular frame to examine their photophysical properties. In continuation of our work, ${ }^{11}$ we herein describe an efficient one-pot synthesis of dibenzofuran, dibenzothiophene and 9-methyl-9H-carbazoles embedded xanthene-1,8diones through the condensation of respective aldehydes with active cyclic1,3-dicarbonyl in presence of PPA-SiO ${ }_{2}$ catalyst. UV-Visible absorption properties are evaluated for these new derivatives.

Initiating the study, dibenzo[ $b, d]$ furan-2-carbaldehyde (2a), 9-methyl-9H-carbazole-3-carbaldehyde (2b) and dibenzo $[b, d]$ thiophene-2-carbaldehyde $(\mathbf{2 c})$ required were synthesized using formylation conditions developed in our laboratory (scheme 1). ${ }^{11 \text { a }}$ For example, the reaction dibenzo $[b, d]$ furan with $\alpha, \alpha$-dichloromethyl methyl ether and $\mathrm{SnCl}_{4}$ at room temperature in dichloromethane gave the required dibenzo[b,d]furan-2carbaldehyde (2a) in $84 \%$ yield. Similarly 9-methyl- $9 H$ carbazole and dibenzo[b,d]thiophene was formylated to obtain 9-methyl-9H-carbazole-3-carbaldehyde (2b) and dibenzo $[b, d]$ thiophene-2-carbaldehyde (2c) respectively in $80 \%$ and $60 \%$ yields. All the aldehydes 2a-c was fully characterized by ${ }^{1} \mathrm{H}$ and ${ }^{13} \mathrm{C}$ NMR spectral analysis and are in agreement with reported data. ${ }^{9,11 a}$

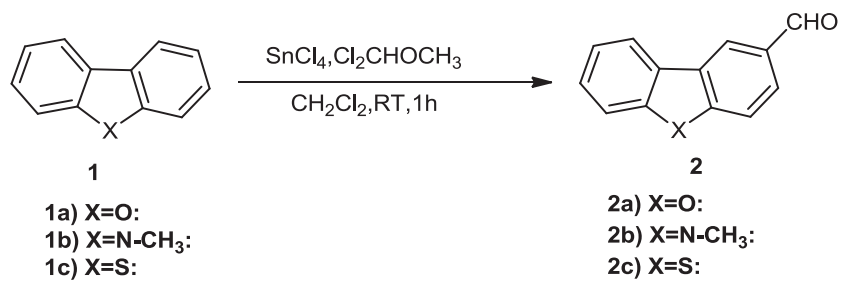

Scheme 1. Preparation of tricyclic heterocyclic aldehydes 2a-c. 
Table 1. Optimization of reaction conditions for a selective reaction between aldehyde 2a and compound $\mathbf{3}$.

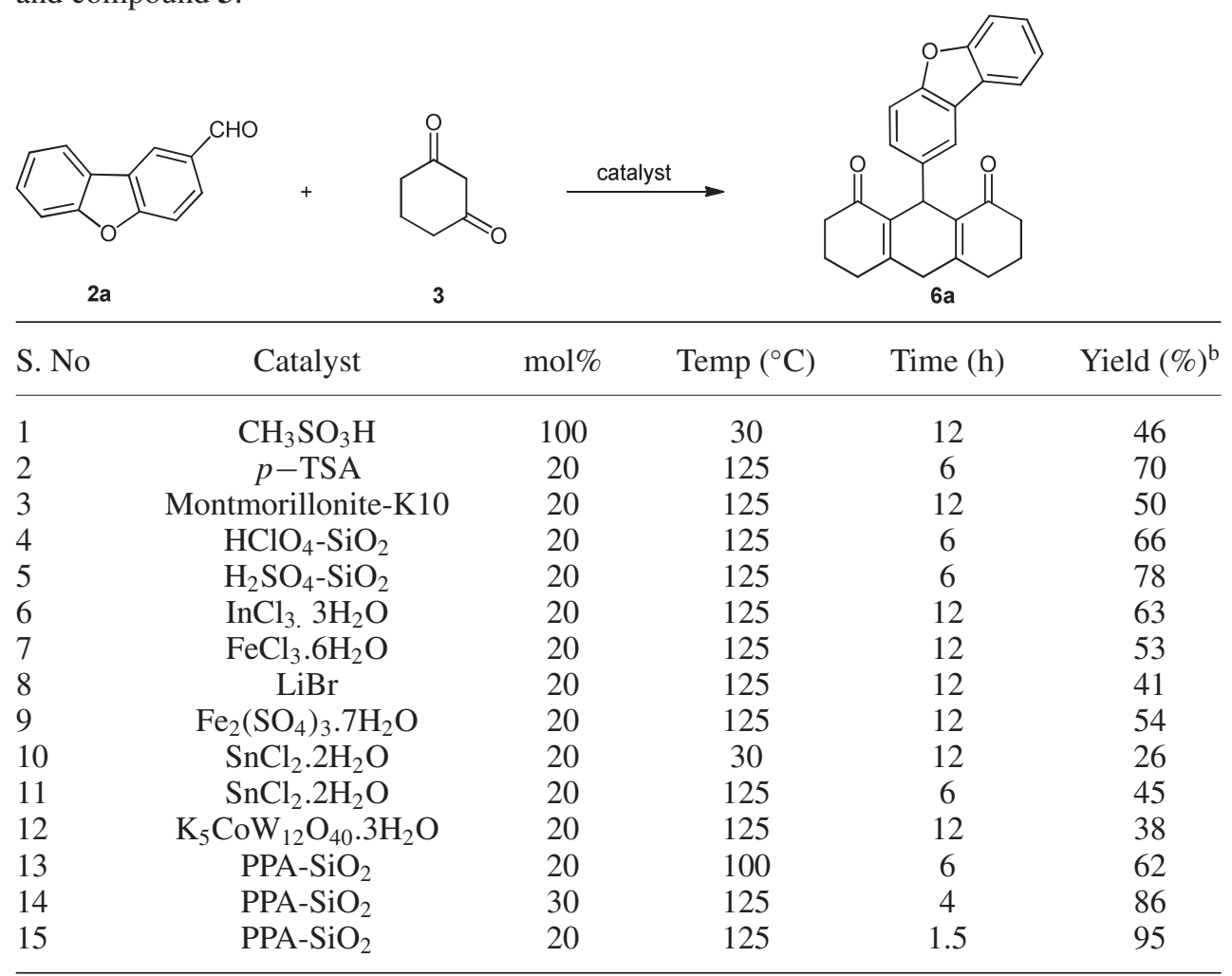

(a) Dibenzo[b,d]furan-2-carbaldehyde (2a) (1 mmol), 1,3-cyclohexane dione 3 (2 mmol), and catalyst were mixed and conducted the reaction.

(b) Isolated yields of $\mathbf{6 a}$

With the required aldehydes $\mathbf{2 a}-\mathbf{c}$ in hand, we now focused on the synthesis of new xanthenedione derivatives through the condensation of aldehydes $\mathbf{2 a - c}$ with different cyclic diketones [1,3-cyclohexanedione (3), dimedone (4) and 5-isopropyl 1,3-cyclohexanedione (5)] in presence of various catalysts and solvents. Initial efforts were focused on the reaction of aldehyde 2a and 1,3-cyclohexanedione (3) with different catalysts $\mathrm{InCl}_{3} .3 \mathrm{H}_{2} \mathrm{O}, \mathrm{FeCl}_{3} \cdot 6 \mathrm{H}_{2} \mathrm{O}, \mathrm{LiBr}, \mathrm{Fe}_{2} \mathrm{SO}_{4} .7 \mathrm{H}_{2} \mathrm{O}$, $\mathrm{SnCl}_{2} \cdot 2 \mathrm{H}_{2} \mathrm{O}$ and $\mathrm{K}_{5} \mathrm{CoW}_{12} \mathrm{O}_{40} .3 \mathrm{H}_{2} \mathrm{O} \mathrm{CH}_{3} \mathrm{SO}_{3} \mathrm{H}, p-\mathrm{TSA}$, montmorillonite- $\mathrm{K} 10, \mathrm{HClO}_{4}-\mathrm{SiO}_{2}, \mathrm{H}_{2} \mathrm{SO}_{4}-\mathrm{SiO}_{2}$. The yields of isolated product xanthenedione $\mathbf{6 a}$ along with reaction temperatures and catalysts used are shown in table 1. Among the catalysts screened, the reaction with $20 \mathrm{~mol} \%$ of PPA-SiO${ }_{2}$ catalyst under solvent-free conditions proceeded efficiently and smoothly in shorter reaction time $(1.5 \mathrm{~h})$ at $125^{\circ} \mathrm{C}$ to give the xanthenedione derivative 6a in very good yield in $95 \%$ (table 1, entry 15).

In order to investigate the general applicability and versatility of this procedure for the synthesis of variety of substituted xanthenedione derivatives, condensation<smiles>[X]c1ccc2[X]c3ccccc3c2c1</smiles>

$2 a, 2 b, 2 c$<smiles>[R]C1([R])CC(=O)CC(=O)C1</smiles>

3; $\mathrm{R}_{1}, \mathrm{R}_{2}=\mathrm{H}$

4; $R_{1}, R_{2}=-M e$

5; $\mathrm{R}_{1}=\mathrm{H}, \mathrm{R}_{2}=$-Isopropyl

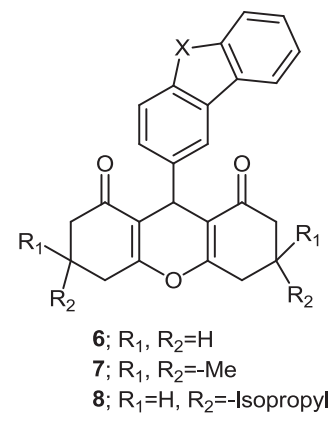

Scheme 2. Synthesis of tricyclic heterocycle tethered xanthenediones $\mathbf{6 a}-\mathbf{c}, 7 \mathbf{a}-\mathbf{c}$ and $\mathbf{8 a}-\mathbf{c}$. 
Table 2. Synthesis of novel dibenzo[b,d]furan, dibenzo[ $[b, d]$ thiophene, 9-methyl-9Hcarbazole embedded xanthene-1,8-diones 6a-c, 7a-c and 8a-c with recyclable PPA-SiO catalyst.

\begin{tabular}{lccccc}
\hline S.No & Aldehyde & diketone & Product $^{\mathrm{a}}$ & Reaction time (h) & Yield (\%) $^{\mathrm{b}}$ \\
\hline 1 & $2 \mathrm{a}$ & 3 & $6 \mathrm{a}$ & 1.5 & 95 \\
2 & $2 \mathrm{a}$ & 4 & $7 \mathrm{a}$ & 2 & 92 \\
3 & $2 \mathrm{a}$ & 5 & $8 \mathrm{a}$ & 1.5 & 94 \\
4 & $2 \mathrm{~b}$ & 3 & $6 \mathrm{~b}$ & 1.5 & 91 \\
5 & $2 \mathrm{~b}$ & 4 & $7 \mathrm{~b}$ & 1.5 & 93 \\
6 & $2 \mathrm{~b}$ & 5 & $8 \mathrm{~b}$ & 2 & 92 \\
7 & $2 \mathrm{c}$ & 3 & $6 \mathrm{c}$ & 1.5 & 93 \\
8 & $2 \mathrm{c}$ & 4 & $7 \mathrm{c}$ & 2 & 94 \\
9 & $2 \mathrm{c}$ & 5 & $8 \mathrm{c}$ & 2 & \\
\hline
\end{tabular}

a) All the reactions were performed without solvent at $125^{\circ} \mathrm{C}$.

b) Yields of isolated products.<smiles>O=C1CCCC2=C1C(c1ccc3oc4ccccc4c3c1)C1=C(CCCC1)O2</smiles>

$6 a$<smiles>Cn1c2ccccc2c2cc(C3C4=C(CCCC4=O)OC4=C3C(=O)CCC4)ccc21</smiles><smiles>O=C1CCCC2=C1C(c1ccc3sc4ccccc4c3c1)C1=C(CCCC1=O)O2</smiles><smiles>CC1(C)CC(=O)C2=C(C1)OC1=C(C(=O)CC(C)(C)C1)C2c1ccc2oc3ccccc3c2c1</smiles><smiles>Cn1c2ccccc2c2cc(C3C4=C(CC(C)(C)CC4=O)OC4=C3C(=O)CC(C)(C)C4)ccc21</smiles>

7b<smiles>CC(C)C1CC(=O)C2=C(C1)OC1=C(C(=O)CC(C(C)C)C(C)C1)C2c1ccc2oc3ccccc3c2c1</smiles><smiles>CC(C)C1CC(=O)C2=C(C1)OC1=C(C(=O)CC(C(C)C)C1)C2c1ccc2c(c1)c1ccccc1n2C</smiles><smiles>CC(C)C1CC(=O)C2=C(C1)OC1=C(C(=O)CC(C(C)C)C1)C2c1ccc2sc3ccccc3c2c1</smiles>

Figure 1. Structures of xanthenedione derivatives 6, 7 and 8 .

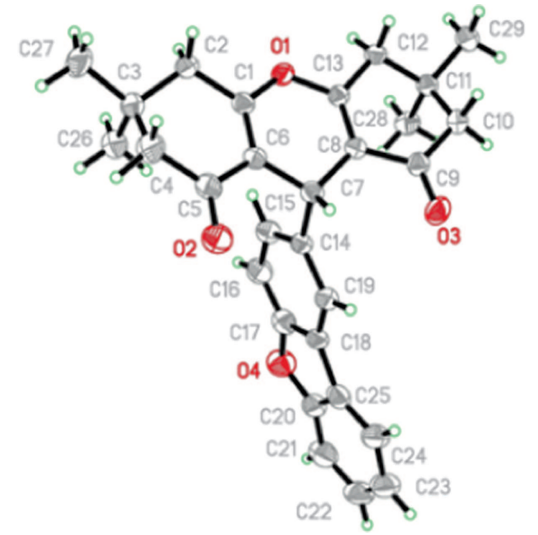<smiles>C=CCCCCCC1=C2CC(C)(C)CC(=O)C2=C2OC3=C(C(=O)CC(C)(C)C3)C(c3ccc4oc5ccccc5c4c3)C12</smiles>

Figure 2. ORTEP diagram of compound 7a.

of aldehydes $\mathbf{2 a}, \mathbf{2 b}$ and $\mathbf{2 c}$ with activated methylene compounds such as cyclohexane-1,3-dione (3); 5,5dimethyl cyclohexane-1,3-dione (4); 5-isopropylcyclo hexane-1,3-dione (5) were examined (scheme 2). All these reactions proceeded to yield the corresponding xanthenediones $\mathbf{6 a}-\mathbf{c}, \mathbf{7} \mathbf{a}-\mathbf{c}$ and $\mathbf{8 a - c}$ in very good yields 
Table 3. Comparison of absorption properties of compounds $6 \mathbf{a}-\mathbf{c}, 7 \mathbf{7}-\mathbf{c}$ and $\mathbf{8 a}-\mathbf{c}$.

\begin{tabular}{lcccc}
\hline & & \multicolumn{3}{c}{$\lambda_{\max }(\mathrm{nm})\left\{\varepsilon_{\max }\left(\mathrm{L} \mathrm{mole} \mathrm{cm}^{-1}\right)\right\}$} \\
\cline { 3 - 4 } S. No & Product & $\mathrm{In} \mathrm{CHCl}_{3}$ & $\mathrm{In} \mathrm{THF}$ & In $\mathrm{CH}_{3}-\mathrm{CN}$ \\
\hline 1 & $6 \mathrm{a}$ & $287.5(29373)$ & $288.5(36398)$ & $287(17437)$ \\
2 & $7 \mathrm{a}$ & $290.5(28880)$ & $289.5(29807)$ & $288(26259)$ \\
3 & $8 \mathrm{a}$ & $289.5(19660)$ & $289(25702)$ & $292(114965)$ \\
4 & $6 \mathrm{~b}$ & $297.5(12824)$ & $293.5(14715)$ & $291.5(24707)$ \\
5 & $7 \mathrm{~b}$ & $289.5(20296)$ & $293(25158)$ & $292(8442)$ \\
6 & $8 \mathrm{~b}$ & $297.5(14221)$ & $293(20512)$ & $285(17270)$ \\
7 & $6 \mathrm{c}$ & $290.5(21491)$ & $286.5(17261)$ & $286(14000)$ \\
8 & $7 \mathrm{c}$ & $291(16719)$ & $286(23009)$ & $294(5300)$ \\
9 & $8 \mathrm{c}$ & $289.5(25673)$ & $302(17000)$ & \\
$10^{\mathrm{a}}$ & 9 & - &
\end{tabular}

a) Product 9 refers to the xanthenedione derivative formed from benzaldehyde and dimedone.

$\sim 90-95 \%$ (table 2). All the products (figure 1) thus obtained were fully characterized by IR, ${ }^{1} \mathrm{H}$ NMR, ${ }^{13} \mathrm{C}$ NMR and Mass spectral analysis.

The structure of xanthenedione 7a was confirmed from its single-crystal X-ray crystallographic analysis (figure 2).

The possible application of these newly synthesized xanthenedione derivatives were investigated for their optical behaviour due to extended conjugation with in-built pyran ring system. The properties of the newly synthesized xanthenediones 6a-c, 7a-c and 8a-c were investigated using JASCO UV-Visible absorption spectrophotometer in dilute solutions of chloroform at a concentration of $10^{-5}$ moles/lit. The data obtained was summarized in table 3 . All the xanthenedione derivatives 6a-c, 7a-c and 8a-c exhibited similar absorption spectras with comparable molar absorptivities at an absorption maxima $\left(\lambda_{\max }\right)$ in the region of $287-291 \mathrm{~nm}$ in chloroform (figure 3 ). This absorption bands could

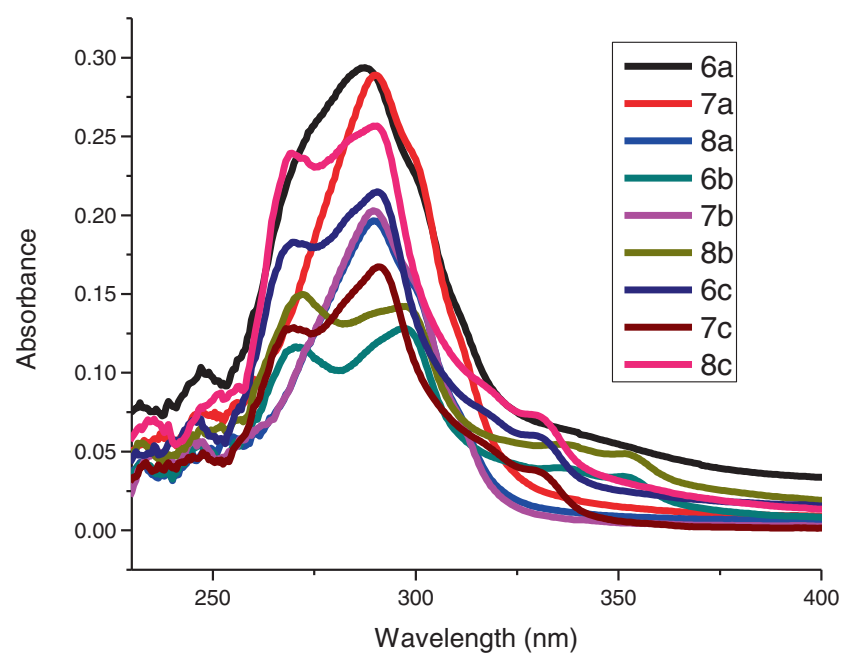

Figure 3. Absorption Spectrum of 6a-c, 7a-c and 8a-c in $\mathrm{CHCl}_{3}$ at a concentration of $10^{-5} \mathrm{M}$. be assigned due to the excitation of $\pi$ electrons existing in the xanthenedione derivatives $\mathbf{6 a - c}, \mathbf{7 a - c}$ and $\mathbf{8 a}-\mathbf{c}$.

Further to study the effect of solvent on the UVVisible absorption properties of xanthenediones, investigations were carried out using various solvents like tetrahydrofuran and acetonitrile and the results were correlated with the absorption properties in chloroform (figure 4-6 and table 3). Among these xanthenediones evaluated, compounds having dibenzo $[b, d]$ furan $6 \mathbf{6}, 7 \mathbf{a}$ and 8a has shown greater molar extinction coefficient than that of the other xanthenedione derivatives $\mathbf{6 b}-\mathbf{c}$, $\mathbf{7 b - c}$, and 8b-c. Moreover observation of the figures 35 , revealed that the xanthenedione derivative $6 \mathbf{a}, 7 \mathbf{a}$ and 8a did not exhibit any solvatochromism. Almost all the compounds have shown greater $\varepsilon_{\max }$ in tetrahydrofuran solvent compared to other solvents used.

When correlated with the known xanthenedione $\mathbf{9}^{12}$ having phenyl ring (i.e., xanthenedione derived from benzaldehyde and dimedone, figure 7), the newly

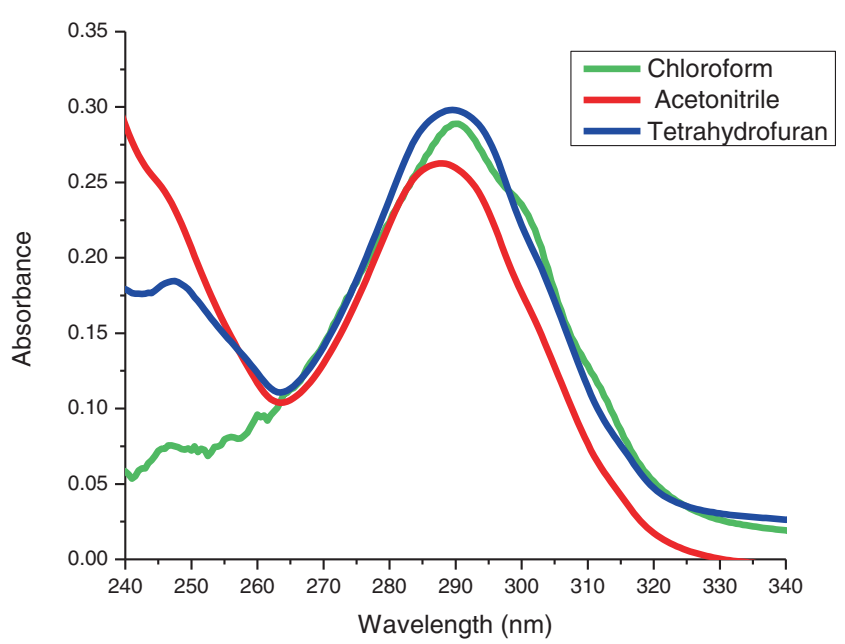

Figure 4. Absorption Spectrum of $7 \mathbf{a}$ in THF, $\mathrm{CH}_{3}-\mathrm{CN}$ and $\mathrm{CHCl}_{3}$. 


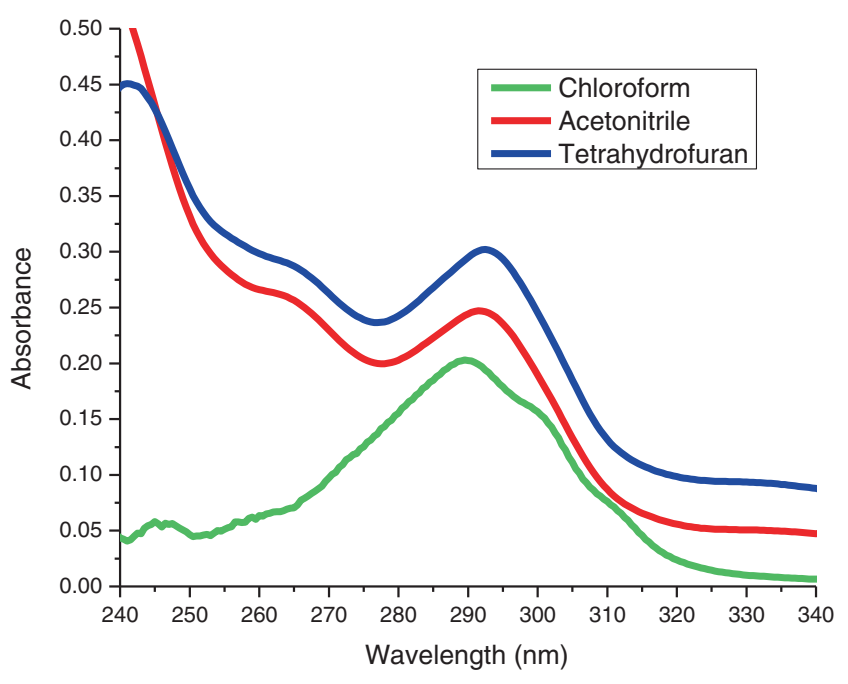

Figure 5. Absorption Spectrum of $\mathbf{7 b}$ in $\mathrm{THF}, \mathrm{CH}_{3}-\mathrm{CN}$ and $\mathrm{CHCl}_{3}$.

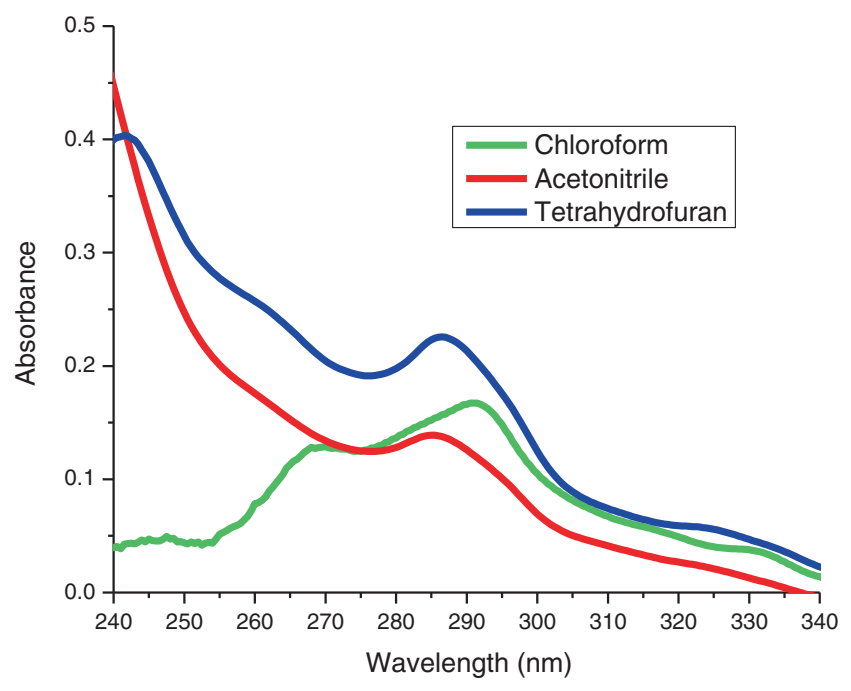

Figure 6. Absorption Spectrum of 7c in THF, $\mathrm{CH}_{3}-\mathrm{CN}$ and $\mathrm{CHCl}_{3}$.

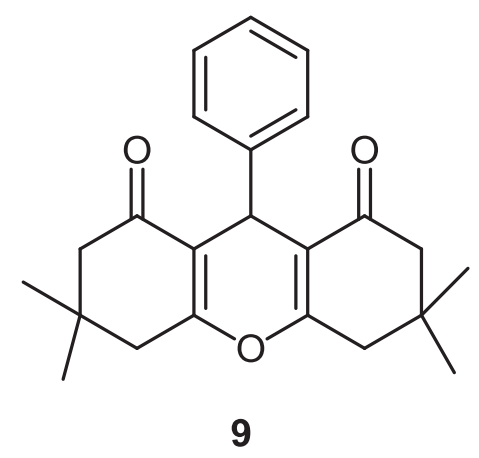

Figure 7. Structure of xanthenedione derivative 9 derived from benzaldehyde and dimedone. synthesized xanthenedione derivatives $\mathbf{6 a - c}, 7 \mathbf{a}-\mathbf{c}$ and 8a-c showed higher molar extinction values $\left(\varepsilon_{\max }\right)$ and lower absorption wavelengths $\left(\lambda_{\max }\right)$. The lowering in the absorption wavelengths may be due to the interference of the non-bonded electrons present on the heteroatom with the extending $\pi$ bonded conjugated electrons which results in increasing the energy gap $\left(E_{g}\right)$ between HOMO and LUMO. ${ }^{13}$

\section{Conclusions}

We have described an efficient one-pot synthesis of novel dibenzofuran, carbazole and dibenzothiophene embodied xanthenediones $(\mathbf{6 a}-\mathbf{c}, \mathbf{7 a - c}$ and $\mathbf{8 a}-\mathbf{c})$ through the condensation of respective aldehydes with cyclic 1,3-diketones in the presence of PPA-SiO${ }_{2}$ catalyst $(20 \mathrm{~mol} \%)$. Absorption spectra of all the newly synthesized xanthenediones $\mathbf{6 a - c}, \mathbf{7 a - c}$ and $\mathbf{8 a}-\mathbf{c}$ suggested that the new compounds have greater molar extinction coefficient values when compared to the xanthenedione formed from benzaldehyde and dimedone.

\section{Supplementary Information}

CCDC 849770 contains Crystallographic data for the compound 7a. This data can be obtained free of charge via http://www.ccdc.cam.ac.uk/datarequest/cif. Scanned copies of ${ }^{1} \mathrm{H},{ }^{13} \mathrm{C}$ and ESI-HRMS spectra of 6a-c, 7a-c and 8a-c are given in electronic supporting information available at www.ias.ac.in/chemsci.

\section{Acknowledgments}

Authors (TY and SK) are thankful to Dr. Lakshmi Kantham, Director and Dr. V. J.Rao, Head, Crop Protection Chemicals Division, IICT, Hyderabad for their continuous support and financial assistance from CSIR projects (NWP0054 and NWO0055). TY (SRF) is thankful to CSIR for fellowship.

\section{References}

1. Omolo J J, Johnson M M, Van-Vuuren S F and De- Koning C B 2011 Bioorg. Med. Chem. Lett. 217085

2. Mulakayala N, Murthy $\mathrm{P}$ V N S, Rambabu D, Aeluri M, Adepu R, Krishna G R, Reddy C M, Prasad K R, Chaitanya M, Kumar C S, Rao M V and Pal M 2012 Bioorg. Med. Chem. Lett. 222186

3. Shadid A K, Shaari K, Abas F, Israf D A, Hamzah A S, Syakroni N, Saha K and Lajis N H 2007 Phytochemistry 682537

4. Fabre P, Cosmetique, Stephane P and Francoise B 2012 Patent: WO2012/4390 A1

5. Khuran J M, Chaudhary A, Lumb A and Nand B 2012 Can. J. Chem. 90739 
6. Menchen S M, Benson S C, Lam J Y L, Zhen W, Sun D, Rosenblum B B, Khan S H and Taing M 2013 US Patent, 6583168. [Chem. Abstr. 2003, 139]

7. Ahmad M, King T A, Ko D K, Cha B H and Lee J 2002 J. Phys. D: Appl. Phys. 351473

8. Knight C G and Stephens T 1989 Biochem. J. 258683

9. Qiao Y, Wei Z, Risko C, Li H, Luc Bredas J, Xu W and Zhu D 2012 J. Mater. Chem. 221313

10. (a) Prado S, Ledeit H, Michel S, Koch M, Darbord J C, Cole S T, Tillequin F and Brodin P 2006 Bioorg. Med. Chem. 14 5423; (b) Kantevari S, Yempala T, Surineni G, Sridhar B, Yogeeswari P and Sriram D 2011 Eur. J. Med. Chem. 46 4827; (c) Zhang F F, Gan L L and Zhou C H 2010 Bioorg. Med. Chem. Lett. 20
1881; (d) Patpi S R, Pulipati L, Yogeeswari P, Sriram D, Jain N, Sridhar B, Murthy R, Devi T A, Kalivendi S V and Kantevari S 2012 J. Med. Chem. 553911

11. (a) Kantevari S, Yempala T, Surineni G, Sridhar B, Yogeeswari P and Sriram D 2011 Eur. J. Med. Chem. 46 4827; (b) Kantevari S, Yempala T, Yogeeswari P, Sriram D and Sridhar B 2011 Bioorg. Med. Chem. Lett. 21 4316; (c) Yempala T, Sriram D, Yogeeswari $\mathrm{P}$ and Kantevari S 2012 Bioorg. Med. Chem. Lett. 22 7426

12. Verma G K, Raghuvanshi K, Verma R K, Dwivedi P and Singh M S 2011 Tetrahedron 673698

13. Bo H, Chan Y, Wei W Q, Hao Z and JianKang Y U 2012 Science China Chemistry $\mathbf{5 5} 1364$ 